\title{
Direct oral anticoagulants for venous thromboembolism prophylaxis in critically ill patients: where do we go from here?
}

Laurent Bertoletti ${ }^{1,2,3}$, Martin Murgier ${ }^{2,4}$ and Henry T. Stelfox ${ }^{5,6,7^{*}}$

(c) 2019 Springer-Verlag GmbH Germany, part of Springer Nature

Venous thromboembolism (VTE) is an important problem and a particular challenge for critically ill patients. First, admission to an intensive care unit (ICU) is as a strong independent risk factor for provoked VTE [1] with an incidence of approximately 9\% [2]. Second, establishing a diagnosis of VTE in critically ill patients is more likely to be delayed or missed than for other patient populations because of comorbid illnesses (e.g., heart failure) and therapies (e.g., sedation) that mask symptoms and signs [1]. Third, critically ill patients diagnosed with VTE experience more anticoagulant therapy-related complications because of increased bleeding risk $[1,3]$. Together these three challenges make effective VTE prophylaxis particularly important for critically ill patients. Epidemiological data suggest that earlier initiation of thromboprophylaxis is associated with reduced incidence of VTE and increased survival [4]. Randomized controlled trials (RCTs) have established the efficacy of heparins in the prevention of VTE in ICU patients [5, 6]. The question remains whether new approaches to prophylaxis can further decrease the risk of VTE without increasing complications.

Direct oral anticoagulants (DOACs) are novel agents with characteristics that make them potentially attractive for VTE prophylaxis. They are easy to use, administered orally (both a strength and limitation), have short halflives, and avoid the risk of heparin-induced thrombocytopenia [7]. Conversely, DOACs are partly metabolized by the liver, eliminated by the kidney, and interact with

\footnotetext{
*Correspondence: tstelfox@ucalgary.ca

${ }^{6}$ Department of Community Health Sciences, University of Calgary, 3280 Hospital Drive NW, Calgary, AB T2N 4Z6, Canada

Full author information is available at the end of the article
}

other medications [7]. Three large RCTs have evaluated the efficacy of VTE prophylaxis regimens that compared longer durations of prophylaxis (range 30-42 days) with DOACs to standard durations of prophylaxis (range 6-14 days) with low molecular weight heparin (LMWH) in patients hospitalized for an acute medical illness. The MAGELLAN trial (8101 patients) reported that rivaroxaban reduced the risk of VTE and increased the risk of bleeding compared to enoxaparin [8]. The ADOPT trial (6528 patients) reported that apixaban was not superior to enoxaparin in preventing VTE and increased the risk of major bleeding [9]. The APEX trial (7513 patients) reported no significant difference between betrixaban and enoxaparin for VTE or major bleeding [10].

In this issue of Intensive Care Medicine, Chi et al. report the results of a post hoc subgroup analysis of 703 patients from the APEX trial who were admitted to the ICU during their hospital stay [11]. The patients included in the analyses were older (mean age $>70$ years) with diagnoses of heart failure, stroke, infection, or respiratory failure and spent approximately 6 days in the ICU and 2 weeks in hospital. Patients with septic shock, severe kidney disease (acute or chronic), recent injuries, and liver dysfunction were excluded. The authors report that at 35-42 days, patients who received betrixaban were significantly less likely to have experienced VTE $(4.27 \%$ vs $7.95 \%, p=0.042$ ) than those who received enoxaparin (Fig. 1). The incidence of major bleeding (1.14\% vs $3.13 \%$, $p=0.07)$ was similar in both groups while non-major bleeding $(2.56 \%$ vs $0.28 \%, p=0.011)$ was significantly more common in patients who received betrixaban. These findings in a subgroup of patients admitted to the ICU are distinctly different from those reported in the primary analyses of the MAGELLAN, ADOPT, and 


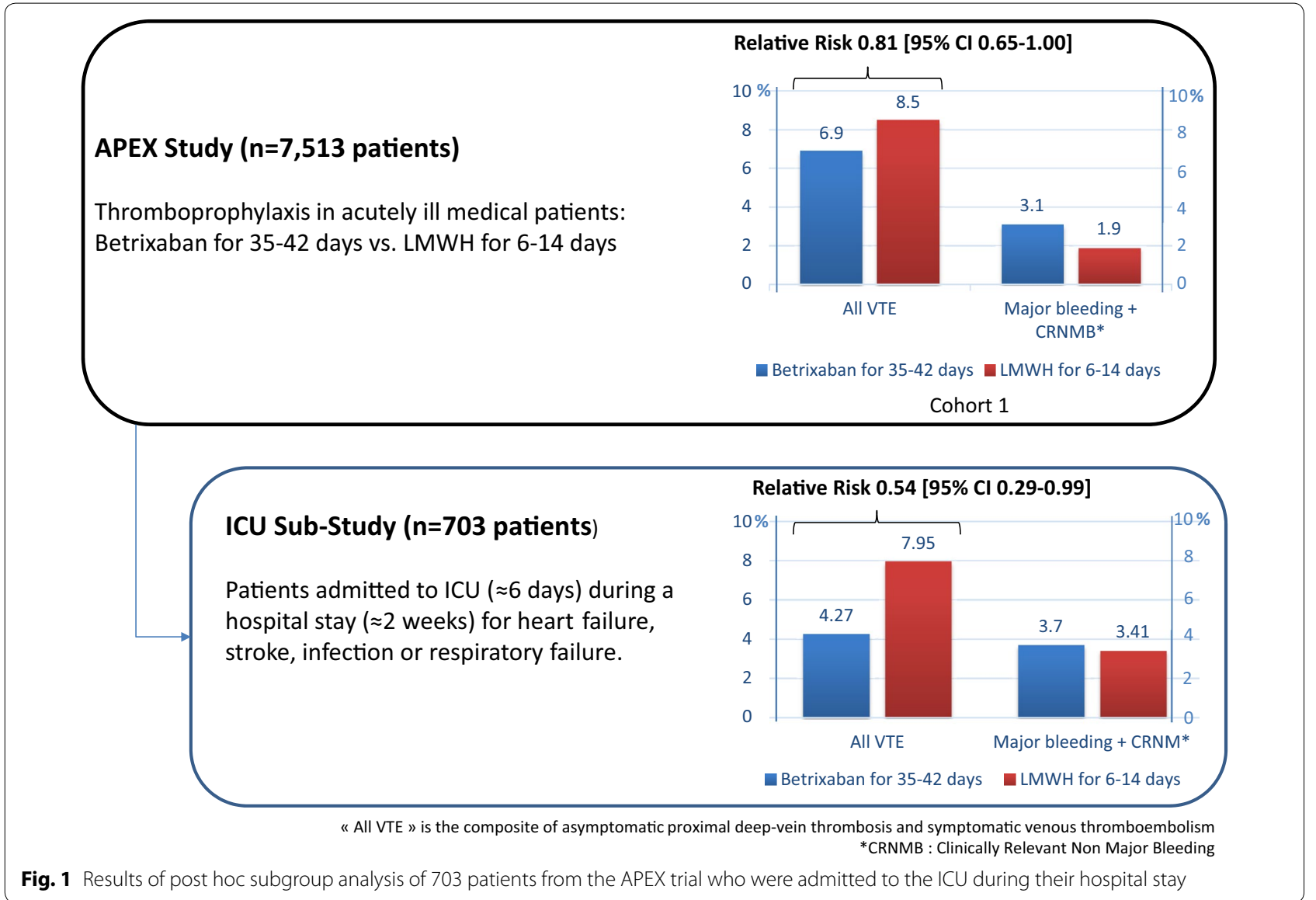

APEX trials and raise the following question: what should clinicians caring for critically ill patients do with this data?

The simple answer is that clinicians should not change their practice. The data presented in the APEX ICU substudy generates an important hypothesis that warrants further evaluation before informing clinical practice. As many of us have experienced, critical care has a recent history of research producing oscillating evidence that has left many clinicians confused and frustrated. The early adoption of promising therapies prior to reproduction of the findings in robust trials while tempting has left critical care medicine in repeated quandaries [12]. It has exposed patients to unnecessary risk (e.g., tight glycemic control), and introduced new care practices that are difficult to de-adopt [13]. As clinicians we want to offer our patients care that affords them the best opportunities to recover from their critical illness. Currently that means continuing with the standard of care for VTE prophylaxis, subcutaneous heparin.

Which form of heparin should clinicians use? That is less clear. A systematic review published in 2015 identified eight RCTs (5567 patients) that compared LMWH with unfractionated heparin (UFH) for VTE prophylaxis in the ICU [2]. The pooled data suggest a $10 \%$ relative lower risk of deep vein thrombosis, pulmonary embolism, major bleeding, or death for LMWH compared to UFH. An economic analysis of the largest trial (PROTECT, 3764 patients) reported LMWH to be more effective with similar or lower costs than UFH [14]. However, a recent implementation science study published in Intensive Care Medicine of 12,342 adult patients admitted to 11 ICUs reported no differences in clinical or economic outcomes when VTE prophylaxis was switched from UFH to LMWH in real-world clinical conditions [15]. Based on the available data, guidelines recommend the use of subcutaneous heparin for thromboprophylaxis, but disagree on the optimal formulation [6].

Where does this leave the use of DOACs for thromboprophylaxis in critically ill patients? The results of the APEX ICU substudy are important because they suggest that there may be value in a definitive evaluation of efficacy. Furthermore, the data raise the possibility that VTE prophylaxis for critically ill patients may need to be considered beyond a patient's hospital stay. So, what question do clinicians need answered to inform their clinical 
practice? The APEX study compared longer duration prophylaxis with betrixaban to standard duration prophylaxis with enoxaparin. The challenge with this approach is that it combines two distinct questions. First, what is the optimal duration of VTE prophylaxis? Second, what is the optimal agent for VTE prophylaxis? Combining both questions into a single study is efficient, but makes it impossible to know whether it is the duration of VTE prophylaxis, the specific thromboprophylaxis agent, or a combination of both that potentially promises better patient outcomes. Clinicians would benefit from future studies that disentangle these questions so that we can be informed as to which agents to use, at which stages of a patient's illness, and for how long.

\section{Author details \\ ${ }^{1}$ Service de Médecine Vasculaire et Thérapeutique, CHU de St-Etienne, Saint-Etienne, France. ${ }^{2}$ INSERM, UMR1059, Equipe Dysfonction Vasculaire et Hémostase, Université Jean-Monnet, 42055 Saint-Etienne, France. ${ }^{3}$ INSERM, CHU Saint-Etienne, CIC-1408, 42055 Saint-Etienne, France. ${ }^{4}$ Service de Médecine Intensive et Réanimation, CHU de St-Etienne, Saint-Etienne, France. ${ }^{5}$ Department of Critical Care Medicine, University of Calgary, Calgary, AB, Canada. ${ }^{6}$ Department of Community Health Sciences, University of Calgary, 3280 Hospital Drive NW, Calgary, AB T2N 4Z6, Canada. ${ }^{7}$ Alberta Health Ser- vices, Calgary, AB, Canada.}

\section{Compliance with ethical standards}

\section{Conflicts of interest}

On behalf of all authors, the corresponding author states that there is no conflict of interest.

\section{Publisher's Note}

Springer Nature remains neutral with regard to jurisdictional claims in published maps and institutional affiliations.

Received: 5 March 2019 Accepted: 18 March 2019

Published online: 25 March 2019

\section{References}

1. Minet C, Potton L, Bonadona A et al (2015) Venous thromboembolism in the ICU: main characteristics, diagnosis and thromboprophylaxis. Crit Care 19:287
2. Alhazzani W, Lim W, Jaeschke RZ et al (2013) Heparin thromboprophylaxis in medical-surgical critically ill patients: a systematic review and metaanalysis of randomized trials. Crit Care Med 41:2088-2098

3. Decousus H, Tapson VF, Bergmann J-F et al (2011) Factors at admission associated with bleeding risk in medical patients: findings from the IMPROVE investigators. Chest 139:69-79

4. Ho KM, Chavan S, Pilcher D (2011) Omission of early thromboprophylaxis and mortality in critically ill patients: a multicenter registry study. Chest 140:1436-1446

5. Duranteau J, Taccone FS, Verhamme P, Ageno W (2018) European guidelines on perioperative venous thromboembolism prophylaxis: intensive care. Eur J Anaesthesiol 35:142-146

6. Schünemann HJ, Cushman M, Burnett AE et al (2018) American Society of Hematology 2018 guidelines for management of venous thromboembolism: prophylaxis for hospitalized and nonhospitalized medical patients. Blood Adv 2:3198-3225

7. Bertoletti L, Ollier E, Duvillard C et al (2017) Direct oral anticoagulants: current indications and unmet needs in the treatment of venous thromboembolism. Pharmacol Res 118:33-42

8. Cohen AT, Spiro TE, Büller HR et al (2013) Rivaroxaban for thromboprophylaxis in acutely ill medical patients. N Engl J Med 368:513-523

9. Goldhaber SZ, Leizorovicz A, Kakkar AK et al (2011) Apixaban versus enoxaparin for thromboprophylaxis in medically ill patients. N Engl I Med 365:2167-2177

10. Cohen AT, Harrington RA, Goldhaber SZ et al (2016) Extended thromboprophylaxis with betrixaban in acutely ill medical patients. N Engl J Med 375:534-544

11. Chi G, Gibson C, Kalayci A et al (2019) Extended-duration betrixaban versus shorter-duration enoxaparin for venous thromboembolism prophylaxis in critically ill medical patients: an APEX trial substudy. Intensive Care Med. https://doi.org/10.1007/s00134-019-05565-6

12. Niven DJ, McCormickTJ, Straus SE et al (2018) Reproducibility of clinical research in critical care: a scoping review. BMC Med 16:26

13. Niven DJ, Rubenfeld GD, Kramer AA, Stelfox HT (2015) Effect of published scientific evidence on glycemic control in adult intensive care units. JAMA Intern Med 175:801-809

14. Fowler RA, Mittmann N, Geerts WH et al (2014) Economic evaluation of the prophylaxis for thromboembolism in critical care trial (E-PROTECT): study protocol for a randomized controlled trial. Trials 15:502

15. Stelfox HT, Brundin-Mather R, Soo A et al (2019) A multicentre controlled pre-post trial of an implementation science intervention to improve venous thromboembolism prophylaxis in critically ill patients. Intensive Care Med 45:211-222 